\title{
Gauge invariance of the color confinement mechanism due to the Abelian dual Meissner effect
}

\author{
Katsuya Ishiguro, ${ }^{* a, d}$ Masayasu Hasegawa, ${ }^{b, d}$ Yoshiaki Koma, ${ }^{c, d}$ Toru Sekido, ${ }^{b}$ and \\ Tsuneo Suzuki $\mathbf{i}^{b, d}$ \\ ${ }^{a}$ Integrated Information Center, Kochi University, Kochi 780-8520, Japan \\ ${ }^{b}$ Institute for Theoretical Physics, Kanazawa University, Kanazawa 920-1192, Japan \\ ${ }^{c}$ Numazu College of Technology, Numazu 410-8501, Japan \\ ${ }^{d}$ RIKEN Nishina Center for Accelerator-Based Science, Wako, Saitama351-0198, JAPAN \\ E-mail: ishiguro@kochi-u.ac.jp masayasu@hep.s.kanazawa-u.ac.jp \\ koma@numazu-ct.ac.jp suzuki@hep.s.kanazawa-u.ac.jp
}

\begin{abstract}
The mechanism of non-Abelian color confinement is studied in SU(2) lattice gauge theory in terms of the Abelian fields and monopoles extracted from non-Abelian link variables without adopting gauge fixing. First, the static quark-antiquark potential and force are computed with the Abelian and monopole Polyakov loop correlators, and the resulting string tensions are found to be identical to the non-Abelian string tension. These potentials also show the scaling behavior with respect to the change of lattice spacing. Second, the profile of the color-electric field between a quark and an antiquark is investigated with the Abelian and monopole Wilson loops. The colorelectric field is squeezed into a flux tube due to monopole supercurrent with the same Abelian color direction. The parameters corresponding to the penetration and coherence lengths show the scaling behavior, and the ratio of these lengths, i.e., the Ginzburg-Landau parameter, indicates that the vacuum type is near the border of the type 1 and type 2 (dual) superconductors. These results are summarized in which the Abelian fundamental charge defined in an arbitrary color direction is confined inside a hadronic state by the dual Meissner effect. As the color-neutral state in any Abelian color direction corresponds to the physical color-singlet state, this effect explains non-Abelian color confinement and supports the existence of a gauge-invariant mechanism of color confinement due to the dual Meissner effect caused by Abelian monopoles.
\end{abstract}

The XXVII International Symposium on Lattice Field Theory

July 26-31, 2009

Peking University, Beijing, China

\footnotetext{
* Speaker.
} 


\section{Introduction}

Color confinement in quantum chromodynamics (QCD) is still an important unsolved problem. 't Hooft [1] and Mandelstam [2] conjectured that the QCD vacuum is a kind of dual superconducting state caused by condensation of magnetic monopoles. The color charges are then confined inside hadrons due to the formation of the color-electric flux tube through the dual Meissner effect.

An interesting idea to realize this conjecture is proposed by 't Hooft [3], such that $\mathrm{SU}(3)$ QCD can be reduced to an Abelian $[\mathrm{U}(1)]^{2}$ theory by adopting a partial gauge fixing, and the colormagnetic monopoles appear according to $\pi_{2}\left(\mathrm{SU}(3) /[\mathrm{U}(1)]^{2}\right)=\mathbb{Z}^{2}$. The role of monopoles for the confinement mechanism is investigated extensively on the lattice by applying Abelian projection in the maximally Abelian (MA) gauge [4, 5], where monopoles are extracted a la DeGrandToussaint [6] as in compact U(1) lattice gauge theory. It is then found that the results strongly support the dual superconducting scenario [7, 8, 9, 10, 11, 12, 13]. The confining properties are dominated by the Abelian fields [7 9] and monopoles [9, 14 15, 16], which are called Abelian dominance and monopole dominance, respectively. The color-electric flux is squeezed by the dual Meissner effect [8, 11, 12, 13]. Moreover monopole condensation is confirmed by the energyentropy balance of the monopole trajectories [16, 17, 18]. These results indicate that there must exist a dual Ginzburg-Landau (GL) type theory as an infrared effective theory of QCD [19 20].

However, there are still serious problems to prove this scenario. First, there are infinite ways of the partial gauge fixing. Since the behavior of the monopoles can depend on the gauge choice, it is not clear if the lattice results in the MA gauge are universal. Second, as the 't Hooft scheme essentially uses the Abelian degrees of freedom, it is not explained how non-Abelian color charges are confined.

Recently, we obtained clear numerical evidences of Abelian dominance and the dual Meissner effect in local unitary gauges [21] and without adopting gauge fixing [22 23] in SU(2) lattice gauge theory, where we have used the DeGrand-Toussaint monopoles [6] as in the MA gauge. These results provide us with the following idea ; there must exist a gauge-invariant mechanism of color confinement due to Abelian monopoles [24 25]. In this paper, we aim to show detailed numerical evidence of how these ideas are realized.

The paper is organized as follows. In Sec. 2, we compute the static quark-antiquark potential and the force with the Abelian and monopole Polyakov loop correlators. In Sec. 3, we investigate the correlation function between the Abelian operators and the Wilson loop. In Sec. 4, we discuss implications of our results, i.e., the Abelian fundamental charge defined in an arbitrary color direction is confined by the dual Meissner effect. The final section 5 is devoted to conclusion and remarks. Details can be found in the publication [22, 23].

\section{Abelian dominance and monopole dominance}

We explain how to extract the Abelian fields and the color-magnetic monopoles from the thermalized non-Abelian SU(2) link variables $U_{\mu}(s)=U_{\mu}^{0}(s)+i \vec{\sigma} \cdot \vec{U}_{\mu}(s)$ where $\vec{\sigma}=\left(\sigma^{1}, \sigma^{2}, \sigma^{3}\right)$ is the Pauli matrix. Abelian link variables in one of the color directions, for example, in the $\sigma^{1}$ direction are defined as $u_{\mu}(s)=\cos \theta_{\mu}(s)+i \sigma^{1} \sin \theta_{\mu}(s)$ where $\theta_{\mu}(s)=\arctan \left(U_{\mu}^{1}(s) / U_{\mu}^{0}(s)\right)$ correspond to the Abelian fields. Without gauge fixing the Abelian fields in any color directions should be 
equivalent. We then define the Abelian field strength tensors as

$$
\Theta_{\mu v}(s)=\theta_{\mu}(s)+\theta_{v}(s+\hat{\mu})-\theta_{\mu}(s+\hat{v})-\theta_{v}(s)=\bar{\Theta}_{\mu v}(s)+2 \pi n_{\mu v}(s),
$$

where $\bar{\Theta}_{\mu v} \in[-\pi, \pi]$ and $n_{\mu v}(s)$ is an integer corresponding to the number of the Dirac strings piercing the plaquette. The monopole currents are then defined by [6]

$$
k_{v}(s)=\frac{1}{4 \pi} \varepsilon_{\mu v \rho \sigma} \partial_{\mu} \bar{\Theta}_{\rho \sigma}(s+\hat{v})=-\frac{1}{2} \varepsilon_{\mu v \rho \sigma} \partial_{\mu} n_{\rho \sigma}(s+\hat{v}) \in \mathbb{Z},
$$

where $\partial_{\mu}$ is regarded as a forward difference.

The Abelian static potential $V_{\mathrm{A}}$ is evaluated from the correlation function of the Abelian Polyakov loop operator

$$
P_{\mathrm{A}}=\exp \left[i \sum_{k=0}^{N_{t}-1} \theta_{4}(s+k \hat{4})\right],
$$

separated at a distance $R$ as $V_{\mathrm{A}}(R)=-\ln \left\langle P_{\mathrm{A}}(0) P_{\mathrm{A}}^{*}(R)\right\rangle /\left(a N_{t}\right)$, where $a$ denotes the lattice spacing and $N_{t}$ the temporal lattice size. We then fit the potential to the usual functional form

$$
V_{\text {fit }}(R)=\sigma R-c / R+\mu
$$

where $\sigma$ denotes the string tension, $c$ the Coulombic coefficient, and $\mu$ the constant. By using the multilevel noise reduction method [26], we showed Abelian dominance such that the Abelian string tension is the same as the non-Abelian one at zero temperature in Ref. [22].

The monopole part of the Polyakov loop operator is extracted as follows. Using the lattice Coulomb propagator $D\left(s-s^{\prime}\right)$, which satisfies $\partial_{v} \partial_{v}^{\prime} D\left(s-s^{\prime}\right)=-\delta_{s s^{\prime}}$ with a forward (backward) difference $\partial_{v}\left(\partial_{v}^{\prime}\right)$, the temporal components of the Abelian fields $\theta_{4}(s)$ are written as

$$
\theta_{4}(s)=-\sum_{s^{\prime}} D\left(s-s^{\prime}\right)\left[\partial_{v}^{\prime} \Theta_{v 4}\left(s^{\prime}\right)+\partial_{4}\left(\partial_{v}^{\prime} \theta_{v}\left(s^{\prime}\right)\right)\right] .
$$

Inserting Eq. (2.5) [and then Eq. (2.1)] to the Abelian Polyakov loop (2.3), we obtain

$$
\begin{aligned}
& P_{\mathrm{A}}=P_{\mathrm{ph}} \cdot P_{\mathrm{mon}}, \\
& P_{\mathrm{ph}}=\exp \left\{-i \sum_{k=0}^{N_{t}-1} \sum_{s^{\prime}} D\left(s+k \hat{4}-s^{\prime}\right) \partial_{v}^{\prime} \bar{\Theta}_{v 4}\left(s^{\prime}\right)\right\}, \\
& P_{\mathrm{mon}}=\exp \left\{-2 \pi i \sum_{k=0}^{N_{t}-1} \sum_{s^{\prime}} D\left(s+k \hat{4}-s^{\prime}\right) \partial_{v}^{\prime} n_{v 4}\left(s^{\prime}\right)\right\} .
\end{aligned}
$$

We call $P_{\mathrm{ph}}$ the photon and $P_{\mathrm{mon}}$ the monopole parts of the Abelian Polyakov loop, respectively. The latter is due to the fact that the Dirac strings $n_{v 4}(s)$ lead to the monopole currents in Eq. (2.2) [6]. Note that the second term of Eq. 2.5) does not contribute to the Abelian Polyakov loop in Eq. 2.3.

We then compute the static potential from the monopole Polyakov loop correlation function. However, since Eq. (2.6) contains the nonlocal Coulomb propagator $D\left(s-s^{\prime}\right)$ and the Polyakov loop is not written as a product of local operators along the time direction, the multilevel method cannot be applied. Without such a powerful noise reduction method, it is hard to measure the Polyakov loop correlation function at zero temperature with the present available computer resource. Thus we consider a finite temperature $T \neq 0$ system in the confinement phase. We set 


\begin{tabular}{c|l|l|c|c}
\hline$\beta$ & $N_{s}^{3} \times N_{t}$ & $a(\beta)(\mathrm{fm})$ & $N_{\text {conf }}$ & $N_{\text {RGT }}$ \\
\hline 2.20 & $24^{3} \times 4$ & $0.211(7)$ & 6000 & 1000 \\
2.35 & $24^{3} \times 6$ & $0.137(9)$ & 4000 & 2000 \\
2.35 & $36^{3} \times 6$ & $0.137(9)$ & 5000 & 1000 \\
2.43 & $24^{3} \times 8$ & $0.1029(4)$ & 7000 & 4000 \\
\hline
\end{tabular}

Table 1: Simulation parameters for the measurement of the static potential and the force from $P_{\mathrm{A}}, P_{\mathrm{ph}}$ and $P_{\text {mon. }} N_{\mathrm{RGT}}$ is the number of random gauge transformations.

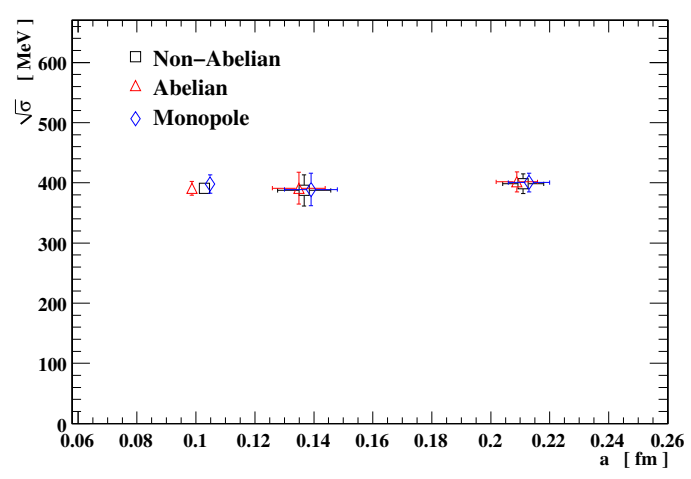

Figure 1: The $a(\beta)$ dependence of the square root of the non-Abelian, Abelian and monopole string tensions for the same temperature $T=0.8 T_{c}$.

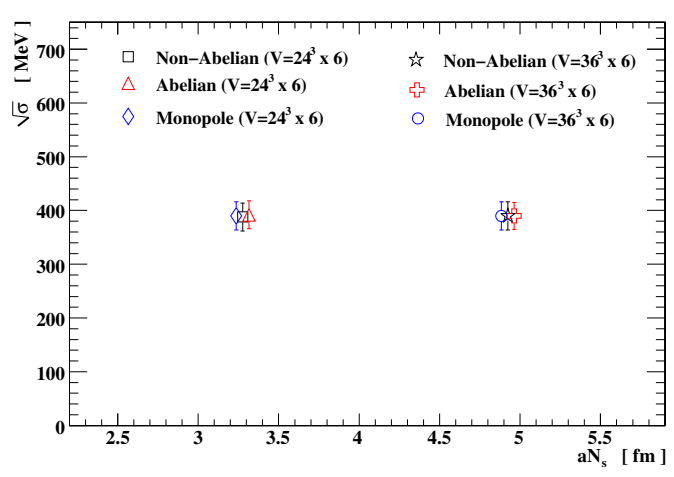

Figure 2: The volume dependence of the square root of the string tensions on the $24^{3} \times 6$ and $36^{3} \times 6$ lattices at $\beta=2.35$.

$T=0.8 T_{c}$. In order to examine the scaling behavior of the potential, we simulate the Wilson action on the $24^{3} \times\left(N_{t}=4,6,8\right)$ lattices. We also investigate the spatial volume dependence of the potential for the $N_{t}=6$ case. Simulation parameters are summarized in Table 1 ,

Since the signal-to-noise ratio of the correlation functions of $P_{\mathrm{A}}, P_{\mathrm{ph}}$, and $P_{\mathrm{mon}}$ are still very small with no gauge fixing, we adopt a new noise reduction method [22]. For a thermalized gauge configuration $U_{\mu}(s)$, we produce many gauge copies $U_{\mu}^{g}(s)$ applying random gauge transformations. Then we compute the operator for each copy and take the average over all copies. The results obtained with this method are gauge averaged, thus, gauge invariant.

We obtain very good signals for the potentials. We fit these potentials to the function $V_{\text {fit }}(R)$ in Eq. (2.4) and extract the string tension and the Coulombic coefficient. Abelian dominance is seen again as in Ref. [22]. Moreover, we observe monopole dominance, i.e., the string tension of the static potential from the monopole Polyakov loop correlation function is identical to that of the non-Abelian static potential, while the potential from the photon Polyakov loop correlation function contains no linear part. It is remarkable that Abelian dominance and monopole dominance for the string tension are almost perfect as explicitly shown in Fig. 1, which also shows the good scaling behavior with respect to the change of lattice spacing. We do not see the volume dependence of the string tension as shown in Fig. 2. These results suggest that although the lattice monopoles defined in Eq. (2.2) are gauge-dependent, they contain physical gauge-invariant pieces responsible for confinement, which show up after taking the gauge average. 


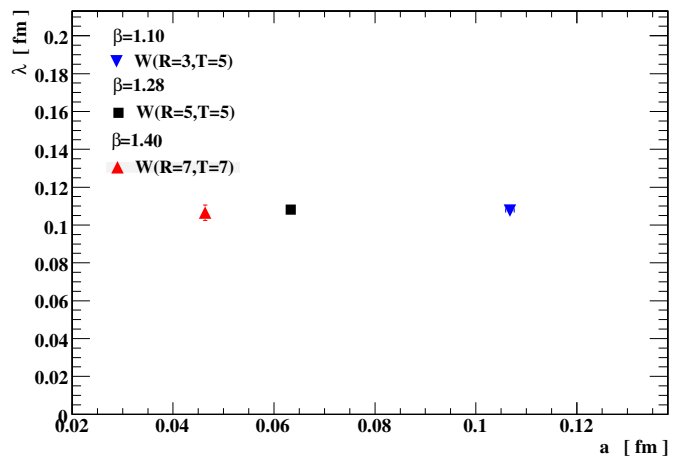

Figure 3: The penetration depth $\lambda$ as a function of lattice spacing $a(\beta)$.

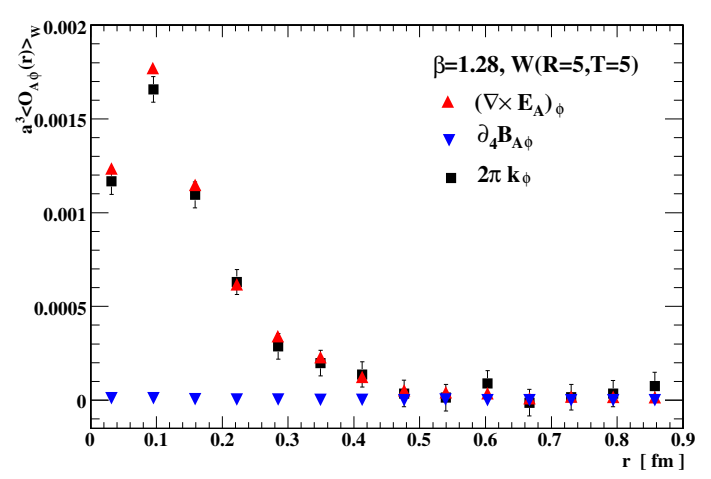

Figure 4: Tests of the dual Ampère law at $\beta=1.28$ for $W(R=5, T=5)$.

\section{The Abelian dual Meissner effect}

We investigate the correlation function [27 28] between a Wilson loop $W$ and a local Abelian operator $\mathscr{O}$ connected by a product of non-Abelian link variables (Schwinger line) $L$,

$$
\langle\mathscr{O}(r)\rangle_{W}=\frac{\left\langle\operatorname{Tr}\left[L W(R, T) L^{\dagger} \sigma^{1} \mathscr{O}(r)\right]\right\rangle}{\langle\operatorname{Tr}[W(R, T)]\rangle} .
$$

We shall use the cylindrical coordinate $(r, \phi, z)$ to parametrize the $q-\bar{q}$ system, where the $z$ axis corresponds to the $q-\bar{q}$ axis and $r$ to the transverse distance. We are interested in the field profile as a function of $r$ on the midplane of the $q-\bar{q}$ system.

In this computation, we employ the improved Iwasaki gauge action [29] with the coupling constants $\beta=1.10$ and 1.28 on the $32^{4}$ lattice, and $\beta=1.40$ on the $40^{4}$ lattice in order to investigate the scaling behavior of the correlation functions with less finite-lattice cutoff effects. We use the Wilson loop $W(3,5)$ at $\beta=1.10, W(5,5)$ at $\beta=1.28$, and $W(7,7)$ at $\beta=1.40$. Note that the physical $q-\bar{q}$ distance is the same $[R=0.32(\mathrm{fm})]$ for these Wilson loops.

We measure all cylindrical components of the color-electric fields $\mathscr{O}(s)=E_{\mathrm{A} i}(s)=\bar{\Theta}_{4 i}(s)$. We find that only $E_{\mathrm{A} z}$ has correlation with the Wilson loop. We then fit $\left\langle E_{\mathrm{A} z}(r)\right\rangle_{W}$ to a function $f(r)=c_{1} \exp (-r / \lambda)+c_{0}$ and find that the profile of $\left\langle E_{\mathrm{A} z}(r)\right\rangle_{W}$ is well described by this functional form, i.e., the color-electric field is exponentially squeezed. The parameter $\lambda$ corresponds to the penetration depth are plotted in Fig. 3 as a function of lattice spacing $a(\beta)$. We find that the penetration depth $\lambda$ shows the good scaling behavior.

To see what squeezes the color-electric field, we study the Abelian (dual) Ampère law derived from the definition of the monopole current in Eq. 2.2],

$$
\vec{\nabla} \times \vec{E}_{\mathrm{A}}=\partial_{4} \vec{B}_{\mathrm{A}}+2 \pi \vec{k}
$$

where $B_{\mathrm{A} i}(s)=(1 / 2) \varepsilon_{i j k} \bar{\Theta}_{j k}(s)$. The correlation of each term with the Wilson loop is evaluated on the same midplane of the $q-\bar{q}$ system as for the profile measurements of the color-electric field. We find that only the azimuthal components are nonvanishing, which are plotted in Fig. 4 Note that if the color-electric field is purely of the Coulomb type, the curl of the electric field is zero. On the 


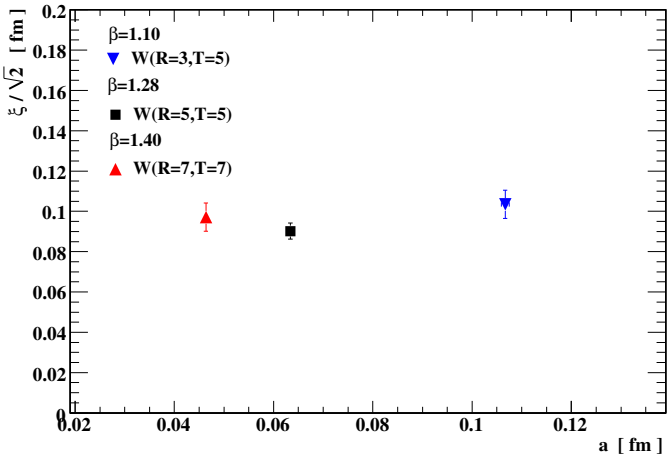

Figure 5: The coherence length $\xi$ as a function of the lattice spacing $a(\beta)$.

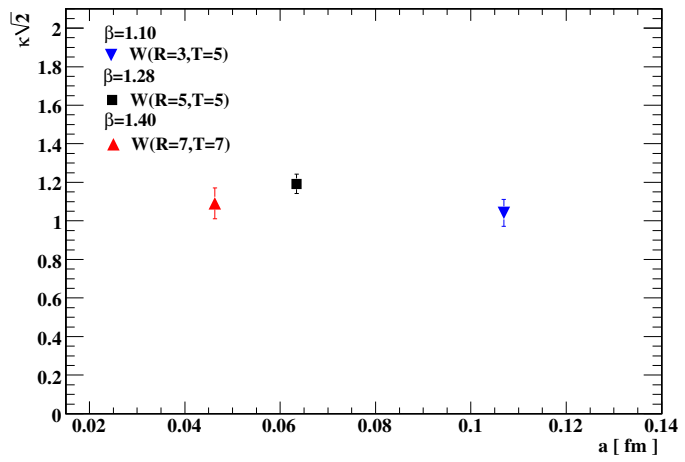

Figure 6: The GL parameters as a function of the lattice spacing $a(\beta)$.

contrary, the curl of the electric field is nonvanishing and is reproduced mostly by the monopole currents. In any case, the dual Ampère law is satisfied, which is a clear signal of the Abelian dual Meissner effect. This result is quite the same as that observed in the MA gauge [12, 13].

Let us estimate the coherence length by evaluating the correlation function between the squared monopole density $\mathscr{O}(s)=k_{\mu}^{2}(s)$ and the Wilson loop [30]. We then fit the profile of $\left\langle k_{\mu}^{2}(r)\right\rangle_{W}$ to the functional form $g(r)=c_{1}^{\prime} \exp (-\sqrt{2} r / \xi)+c_{0}^{\prime}$, where the parameter $\xi$ corresponds to the coherence length. The coherence length shows the scaling behavior as demonstrated in Fig. 5 as a function of lattice spacing $a(\beta)$.

Taking the ratio of the penetration depth and the coherence length, the GL parameter $\sqrt{2} \kappa=$ $\lambda / \xi$ can be estimated, which characterizes the type of the superconducting vacuum. The results are plotted in Fig. 6 against lattice spacing $a(\beta)$. We find that the GL parameter shows the scaling behavior and the value is about 1 . This means that the vacuum type is near the border between the types 1 and 2 dual superconductors. However, we note that the physical spatial size of the Wilson loop used in the present simulations is still small $[R=0.32(\mathrm{fm})]$. Clearly, further quantitative studies with larger Wilson loops are needed to determine the definite value.

\section{Non-Abelian color confinement}

Let us consider what is induced from the above numerical results. Since gauge fixing is not applied in these computations, Abelian fields in any color directions are equivalent. Thus, our result is interpreted as that the color-electric fields in all color directions are squeezed and the Abelian (monopole) string tensions in all color directions are the same as the non-Abelian string tension. This indicates that QCD contains a gauge-invariant Abelian mechanism of confinement which is not related to the specific gauge fixing. Namely Abelian monopoles in three color directions are condensed in the vacuum of the confinement phase of $S U(2)$ QCD.

Let us denote quark fields having charge $1 / 2$ and $-1 / 2$ in the $\sigma_{3}$ direction, respectively, as $u_{3}$ and $d_{3}$. Then local mesonic states, $u_{3} \bar{u}_{3}$ and $d_{3} \bar{d}_{3}$, are Abelian color neutral in the $\sigma_{3}$ direction. Consider next

$$
u_{1}=\frac{u_{3}+d_{3}}{\sqrt{2}}, \quad d_{1}=\frac{u_{3}-d_{3}}{\sqrt{2}}, \quad u_{2}=\frac{i u_{3}+d_{3}}{\sqrt{2}}, \quad d_{2}=\frac{i u_{3}-d_{3}}{\sqrt{2}} .
$$


$u_{1}$ and $d_{1}\left(u_{2}\right.$ and $\left.d_{2}\right)$ are quark fields having charge $1 / 2$ and $-1 / 2$ in the $\sigma_{1}\left(\sigma_{2}\right)$ direction. Using these expressions, the quark-gluon coupling term is written as

$$
\begin{aligned}
\bar{\psi} \gamma^{\mu} \frac{\sigma^{a}}{2} \psi A_{\mu}^{a}= & \frac{1}{2}\left(\bar{u}_{3} \gamma_{\mu} d_{3}+\bar{d}_{3} \gamma_{\mu} u_{3}\right) A_{\mu}^{1}-i \frac{1}{2}\left(\bar{u}_{3} \gamma_{\mu} d_{3}-\bar{d}_{3} \gamma_{\mu} u_{3}\right) A_{\mu}^{2} \\
& +\frac{1}{2}\left(\bar{u}_{3} \gamma_{\mu} u_{3}-\bar{d}_{3} \gamma_{\mu} d_{3}\right) A_{\mu}^{3} \\
= & \frac{1}{2}\left(\bar{u}_{1} \gamma_{\mu} u_{1}-\bar{d}_{1} \gamma_{\mu} d_{1}\right) A_{\mu}^{1}+\frac{1}{2}\left(\bar{u}_{2} \gamma_{\mu} u_{2}-\bar{d}_{2} \gamma_{\mu} d_{2}\right) A_{\mu}^{2} \\
& +\frac{1}{2}\left(\bar{u}_{3} \gamma_{\mu} u_{3}-\bar{d}_{3} \gamma_{\mu} d_{3}\right) A_{\mu}^{3},
\end{aligned}
$$

where the first equation (4.1) is expressed in terms of $u_{3}$ and $d_{3}$ alone. Consider local mesonic states $u_{1} \bar{u}_{1}$ and $d_{1} \bar{d}_{1}\left(u_{2} \bar{u}_{2}\right.$ and $\left.d_{2} \bar{d}_{2}\right)$ which are Abelian color neutral in the $\sigma_{1}\left(\sigma_{2}\right)$ direction. When we look at the states $u_{1} \bar{u}_{1}$ and $d_{1} \bar{d}_{1}$ in the $\sigma_{3}$ direction, they are written as the sum of color-neutral and color-charged states:

$$
u_{1} \bar{u}_{1}=\frac{1}{2}\left(u_{3} \bar{u}_{3}+d_{3} \bar{d}_{3}+u_{3} \bar{d}_{3}+d_{3} \bar{u}_{3}\right), d_{1} \bar{d}_{1}=\frac{1}{2}\left(u_{3} \bar{u}_{3}+d_{3} \bar{d}_{3}-u_{3} \bar{d}_{3}-d_{3} \bar{u}_{3}\right) .
$$

The same observation applies to the color-neutral states $u_{2} \bar{u}_{2}$ and $d_{2} \bar{d}_{2}$ in the $\sigma_{2}$ direction. However, we find that

$$
u_{1} \bar{u}_{1}+d_{1} \bar{d}_{1}=u_{2} \bar{u}_{2}+d_{2} \bar{d}_{2}=u_{3} \bar{u}_{3}+d_{3} \bar{d}_{3},
$$

are Abelian color neutral in all color directions. The state (4.4) is nothing but the non-Abelian color singlet state.

This example tells us that the Abelian color-neutral state in any color directions corresponds to the physical non-Abelian color-singlet state. Hence, the confinement of non-Abelian color charges can be explained in terms of the Abelian dual Meissner effect due to Abelian monopoles.

\section{Concluding remarks}

We make some concluding remarks. The Abelian gauge fields extracted from the thermalized non-Abelian link fields contain originally topological monopoles responsible for the confinement mechanism of non-Abelian color charges even in the continuum limit. Our results presented in this paper are almost the same as those obtained in the maximally Abelian gauge. This suggests that the MA gauge fixing is the easiest method to extract the physical ingredients of the monopoles, since we do not need very precise time-consuming simulations in the MA gauge as done here.

\section{Acknowledgments}

The numerical simulations of this work were done using RSCC in RIKEN and SX-8 computer at RCNP of Osaka University. The authors would like to thank RIKEN and RCNP for their support of computer facilities. The authors are also supported by JSPS and DFG under the Japan-Germany Research Cooperative Program. Y.K. is partially supported by the Ministry of Education, Science, Sports and Culture, Japan, Grant-in-Aid for Young Scientists (B) (No.20740149). 


\section{References}

[1] G. 't Hooft, in Proceedings of the EPS International, edited by A. Zichichi, (Editrice Compositori, Bologna, 1976)p. 1225.

[2] S. Mandelstam, Phys. Rept. 23, 245 (1976).

[3] G. 't Hooft, Nucl. Phys. B190, 455 (1981).

[4] A. S. Kronfeld, M. L. Laursen, G. Schierholz, and U. J. Wiese, Phys. Lett. B198, 516 (1987).

[5] A. S. Kronfeld, G. Schierholz, and U. J. Wiese, Nucl. Phys. B293, 461 (1987).

[6] T. A. DeGrand and D. Toussaint, Phys. Rev. D22, 2478 (1980).

[7] T. Suzuki, Nucl. Phys. Proc. Suppl. 30, 176 (1993).

[8] V. Singh, D. A. Browne, and R. W. Haymaker, Phys. Lett. B306, 115 (1993).

[9] S. Ejiri, S. Kitahara, T. Suzuki, and K. Yasuta, Phys. Lett. B400, 163 (1997).

[10] M. N. Chernodub and M. I. Polikarpov, (1997), [hep-th/9710205].

[11] G. S. Bali, C. Schlichter, and K. Schilling, Prog. Theor. Phys. Suppl. 131, 645 (1998).

[12] Y. Koma, M. Koma, E.-M. Ilgenfritz, T. Suzuki, and M. I. Polikarpov, Phys. Rev. D68, 094018 (2003).

[13] Y. Koma, M. Koma, E.-M. Ilgenfritz, and T. Suzuki, Phys. Rev. D68, 114504 (2003).

[14] J. D. Stack, S. D. Neiman, and R. J. Wensley, Phys. Rev. D50, 3399 (1994).

[15] H. Shiba and T. Suzuki, Phys. Lett. B333, 461 (1994).

[16] H. Shiba and T. Suzuki, Phys. Lett. B351, 519 (1995).

[17] S. Kato, N. Nakamura, T. Suzuki, and S. Kitahara, Nucl. Phys. B520, 323 (1998).

[18] K. Ishiguro, T. Suzuki, and T. Yazawa, JHEP 01, 038 (2002).

[19] Z. F. Ezawa and A. Iwazaki, Phys. Rev. D25, 2681 (1982).

[20] S. Maedan and T. Suzuki, Prog. Theor. Phys. 81, 229 (1989).

[21] T. Sekido, K. Ishiguro, Y. Koma, Y. Mori, and T. Suzuki, Phys. Rev. D76, 031501 (2007).

[22] T. Suzuki, K. Ishiguro, Y. Koma, and T. Sekido, Phys. Rev. D77, 034502 (2008).

[23] T. Suzuki, M. Hasegawa, K. Ishiguro, Y. Koma, and T. Sekido, Phys. Rev. D80, 054504 (2009).

[24] J. M. Carmona, M. D’Elia, A. Di Giacomo, B. Lucini, and G. Paffuti, Phys. Rev. D64, 114507 (2001).

[25] P. Cea and L. Cosmai, Phys. Rev. D62, 094510 (2000).

[26] M. Lüscher and P. Weisz, JHEP 09, 010 (2001).

[27] P. Cea and L. Cosmai, Phys. Rev. D52, 5152 (1995).

[28] A. Di Giacomo, M. Maggiore, and S. Olejnik, Phys. Lett. B236, 199 (1990).

[29] Y. Iwasaki, Nucl. Phys. B258, 141 (1985).

[30] M. N. Chernodub et al., Phys. Rev. D72, 074505 (2005). 